\title{
SEARCH FOR LORENTZ VIOLATION IN A SHORT-RANGE GRAVITY EXPERIMENT
}

\author{
D. BENNETT, V. SKAVYSH, AND J. LONG* \\ Physics Department, Indiana University \\ 727 E. Third St., Bloomington, IN 47408, USA \\ *E-mail: jcl@indiana.edu
}

\begin{abstract}
An experimental test of the Newtonian inverse square law at short range has been used to set limits on Lorentz violation in the pure gravity sector of the Standard-Model Extension. On account of the planar test mass geometry, nominally null with respect to $1 / r^{2}$ forces, the limits derived for the SME coefficients of Lorentz violation are on the order $\bar{s}^{J K} \sim 10^{4}$.
\end{abstract}

\section{Introduction}

Local Lorentz invariance is at the foundation of both the Standard Model of particle physics and General Relativity, but is generally poorly tested for the latter theory. Violation of Lorentz symmetry would break the isotropy of spacetime, permitting the vacuum to fill with 'background' fields that have a preferred direction. Interaction of the test masses in a terrestrial gravity experiment with these fields could result in a sidereal modulation of the force between the masses, thus providing a test of Lorentz invariance in gravity.

A quantitative description of Lorentz violation consistent with local field theory is given by the Standard-Model Extension (SME), which has recently been expanded to include gravitational effects. ${ }^{1}$ The expression for the potential between two test masses $m_{1}$ and $m_{2}$ in the 'pure gravity' sector of the SME is given by:

$$
V=-\frac{G m_{1} m_{2}}{\left|\vec{x}_{1}-\vec{x}_{2}\right|}\left(1+\frac{1}{2} \hat{x}^{\hat{j}} \hat{x}^{\hat{k}} \bar{s}^{\hat{j} \hat{k}}\right)
$$

where $\vec{x}_{1}-\vec{x}_{2}$ is the vector separating $m_{1}$ and $m_{2}, \hat{x}^{\hat{i}}$ is the projection of the unit vector along $\vec{x}_{1}-\vec{x}_{2}$ in the $i$ th direction, and $\bar{s}^{\hat{j} \hat{k}}$ is a set of 9 dimensionless coefficients for Lorentz violation in the standard laboratory 
frame. ${ }^{2}$ Motivated by Ref. 2, this report presents a test of Eq. (1) in a laboratory gravity experiment.

\section{The Indiana short-range experiment}

The Indiana experiment is optimized for sensitivity to possible new forces in nature at short range, which in turn could arise from new elementary particles or even extra spacetime dimensions. ${ }^{3,4}$ It is described in detail elsewhere $;^{5-7}$ here we concentrate on the essential features. To attain sensitivity at short range, the test masses must in general be scaled to that range, so as not to be overwhelmed by Newtonian forces at larger scales.

The experiment is illustrated in Fig. 1. The test masses consist of $250 \mu \mathrm{m}$ thick planar tungsten oscillators, separated by a gap of $100 \mu \mathrm{m}$, with a stiff conducting shield in between them to suppress electrostatic and acoustic backgrounds. The planar geometry concentrates as much mass as possible at the scale of interest, and is nominally null with respect to $1 / r^{2}$ forces. This is effective in suppressing the Newtonian background relative to new short-range effects, but has detrimental consequences for testing the pure gravity sector of the SME. The force-sensitive 'detector' mass is driven by the force-generating 'source' mass at a resonance near $1 \mathrm{kHz}$, placing a heavy burden on vibration isolation. The $1 \mathrm{kHz}$ operation is chosen since at this frequency it is possible to construct a simple, passive vibration isolation system. The entire apparatus is enclosed in a vacuum chamber and operated at $10^{-7}$ torr to further reduce the acoustic coupling. Detector oscillations are read out with a capacitive transducer and JFET amplifier.

This design has proven effective for suppressing all background forces to the extent that the only effect observed is thermal noise due to dissipation in the detector mass. After a run over the course of several days in 2002, this experiment set the strongest limits on new forces of nature between 10 and $100 \mu \mathrm{m} .{ }^{6}$ The experiment has since been optimized to explore gaps below $50 \mu \mathrm{m}$; operation at the thermal noise limit has recently been demonstrated but with limited statistics.

\section{Lorentz violating force for planar geometry}

Analysis of the 2002 data for evidence of Lorentz violation requires a theoretical expression for the Lorentz violating force for the particular test mass geometry. The $j$ th component of the differential force corresponding to the 


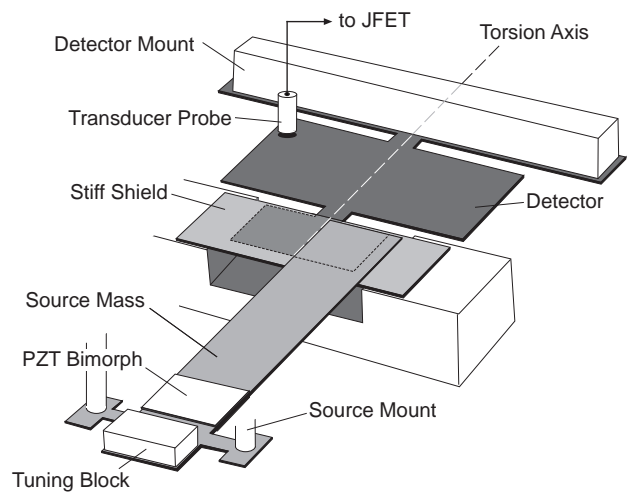

Fig. 1. Central components of Indiana short-range experiment, to scale (the long dimension of the detector mount is approximately $2.5 \mathrm{~cm}$.)

modified potential in Eq. (1) is given by:

$$
d F^{\hat{j}}=G d m_{1} d m_{2}\left(-\frac{x^{\hat{j}}}{x^{3}}-\frac{3}{2} \frac{x^{\hat{j}}}{x^{5}} x^{\hat{j}} x^{\hat{k}} \bar{s}^{\hat{i} \hat{k}}+\frac{x^{\hat{k}} \bar{s}^{\hat{j} \hat{k}}}{x^{3}}\right),
$$

where $x^{\hat{j}}$ and $x^{\hat{k}}$ can be understood as components of the unit vector pointing from a differential mass element $d m_{1}$ in the source mass to a corresponding element $d m_{2}$ in the detector. The SME coefficients in the lab frame $\bar{s}^{\hat{j} \hat{k}}$ are related to the coefficients in the Sun-centered celestial equatorial frame $\bar{s}^{J K}$ by $\bar{s}^{\hat{j} \hat{k}}=R^{T} \bar{s}^{J K} R$, where the matrix $R$ is given by Eq. (C1) of Ref. 8 .

The detector is a constrained mechanical oscillator with distributed mass, so the experiment is sensitive to the force density averaged over the free modal displacement:

$$
F=\frac{1}{|z \max |} \int_{D} d^{3} \vec{x} \vec{z}^{F}(\vec{x}) \cdot \vec{f}(\vec{x}) .
$$

Here, the modal displacement $\vec{z}^{F}(\vec{x})$ is derived from a finite-element model of the detector mass with overall normalization factor $\left|z_{\max }\right|$, and the components of the force density $\vec{f}(\vec{x})$ are given by Eq. (2) above.

Equation (3) is evaluated by Monte Carlo integration with the SME coefficients expressed in the Sun-centered frame and the geometrical parameters listed in Table 2 of Ref. 5. As the experiment is done on resonance, the Monte Carlo algorithm computes the Fourier amplitude of Eq. (3) averaged over a complete cycle of the source mass oscillation, taking into account the measured source mass curvature and mode shape. The result 
can be expressed as a Fourier series of the time dependence,

$$
F=C_{1}+C_{2} \sin \left(\omega_{\oplus} T\right)+C_{3} \cos \left(\omega_{\oplus} T\right)+C_{4} \sin \left(2 \omega_{\oplus} T\right)+C_{5} \cos \left(2 \omega_{\oplus} T\right),
$$

where $\omega_{\oplus}$ is the Earth's sidereal rotation frequency, and the time $T$ is measured in the Sun-centered frame. The $C_{k}$ are functions of the SME coefficients and test mass geometry (and the laboratory colatitude angle) and are given explicitly in Table 1. The statistical errors from the Monte Carlo on the numerical coefficient of each $\bar{s}^{J K}$ term in Table 1 are at most a few percent and ignored for the purpose of computing preliminary limits on the SME coefficients.

Table 1. Signal components for the Indiana short range gravity experiment (when located at colatitude $\chi=0.87$ in 2002).

\begin{tabular}{cr}
\hline \hline Component & Amplitude $\left(\times 10^{-16} \mathrm{~N}\right)$ \\
\hline$C_{1}$ & $1.12 \bar{s}^{X X}+0.00 \bar{s}^{X Y}-7.78 \bar{s}^{X Z}-3.48 \bar{s}^{Y Y}+0.00 \bar{s}^{Y Z}-0.21 \bar{s}^{Z Z}$ \\
$C_{2}$ & $0.07 \bar{s}^{X X}+0.76 \bar{s}^{X Y}+0.02 \bar{s}^{X} Z+0.00 \bar{s}^{Y Y}-0.64 \bar{s}^{Y Z}-0.07 \bar{s}^{Z Z}$ \\
$C_{3}$ & $-0.49 \bar{s}^{X X}+0.11 \bar{s}^{X Y}-0.17 \bar{s}^{X} Z+0.00 \bar{s}^{Y Y}-0.09 \bar{s}^{Y} Z+0.49 \bar{s}^{Z} Z$ \\
$C_{4}$ & $0.06 \bar{s}^{X X}-0.08 \bar{s}^{X Y}+0.15 \bar{s}^{X} Z-0.16 \bar{s}^{Y Y}-0.10 \bar{s}^{Y Z}-0.09 \bar{s}^{Z Z}$ \\
$C_{5}$ & $0.03 \bar{s}^{X X}+0.20 \bar{s}^{X Y}+0.06 \bar{s}^{X Z}-0.06 \bar{s}^{Y Y}+0.24 \bar{s}^{Y} Z+0.04 \bar{s}^{Z} Z$ \\
\hline
\end{tabular}

\section{Limits on coefficients for Lorentz violation}

A preliminary analysis of the 2002 data set (incorporating statistical errors only) for signals of Lorentz violation has been completed, following Ref. 9. In this analysis, the time stamps in the data are extracted and offset relative to the effective $T_{0}$ in the Sun-centered frame (solar noon on the 2002 vernal equinox). The results are shown in Fig. 2 of Ref. 7. The discrete Fourier transforms of the data at each frequency component of the signal $\left(0, \omega_{\oplus}, 2 \omega_{\oplus}\right)$ are computed, with errors. Gaussian probability distributions for the experiment at each signal frequency component are constructed, using the difference between the Fourier transforms and the predicted signals (Table 1) as the means. As in Ref. 9, the overlap of the Fourier components characteristic of the finite data set (Fig. 2 of Ref. 7) is quantified by scaling each term of the predicted signal with the element of a covariance matrix formed from the time stamps of the data. Finally, an overall probability distribution for the experiment is constructed from the product of the component distributions. Means and errors of particular $\bar{s}^{J K}$ (for example, $\left.\bar{s}^{X X}\right)$ are then computed by integration of the distribution over all 
$\bar{s}^{J K}$ except $\bar{s}^{X X}$. Results for each $\bar{s}^{J K}$ (except one) are shown in Table 2. This procedure is sufficient to determine the SME coefficients and account

Table 2. Limits on pure-gravity sector SME Lorentz violation coefficients from the Indiana short-range gravity experiment.

\begin{tabular}{cr}
\hline \hline Coefficient & Mean and error $(2 \sigma)$ \\
\hline $\bar{s}^{X X}$ & $(-0.04 \pm 4.90) \times 10^{4}$ \\
$\bar{s}^{X Y}$ & $(-0.07 \pm 6.12) \times 10^{4}$ \\
$\bar{s}^{X Z}$ & $(-0.01 \pm 2.56) \times 10^{3}$ \\
$\bar{s}^{Y} Z$ & $(-0.06 \pm 5.83) \times 10^{4}$ \\
$\bar{s}^{Z Z}$ & $(0.08 \pm 6.68) \times 10^{4}$ \\
\hline
\end{tabular}

for their correlations, as long as the number of signal components equals or exceeds the number of coefficients in Table 1, which is not the case; for this analysis, $\bar{s}^{Y Y}$, to which the experiment is apparently least sensitive, has been set to zero.

The limits are on the order of $10^{3}-10^{4}$. This is as expected, since, as pointed out in Ref. 2, Lorentz violation leads to a misalignment of the force associated with Eq. (1) relative to the vector $\vec{x}_{1}-\vec{x}_{2}$, however the $1 / r^{2}$ behavior of the force is preserved. The planar test mass geometry renders the Indiana experiment insensitive to $1 / r^{2}$ forces (about $10^{3}$ times gravitational strength at $10 \mu \mathrm{m}$ ) which is directly reflected in the limits on the SME coefficients.

\section{Acknowledgments}

The authors would like to thank H. Muller for useful discussions of Ref. 9.

\section{References}

1. V.A. Kostelecký, Phys. Rev. D 69, 105009 (2004).

2. Q.G. Bailey and V.A. Kostelecký, Phys. Rev. D 74, 045001 (2006).

3. E. Adelberger et al., Ann. Rev. Nucl. Part. Sci. 53, 77 (2003).

4. S. Dimopoulos and A.A. Gearci, Phys. Rev. D 68, 124021 (2003).

5. J.C. Long et al., arXiv:hep-ph/0210004.

6. J.C. Long et al., Nature 421, 922 (2003).

7. W.A. Jensen, S.M. Lewis, and J.C. Long, in V. A. Kostelecký, ed., Proceedings of the Fourth Meeting on CPT and Lorentz Symmetry, World Scientific, Singapore, 2008.

8. V.A. Kostelecký and M. Mewes, Phys. Rev. D 66, 056005 (2002).

9. K.-Y. Chung et al., Phys. Rev. D 80, 016002 (2009). 